\title{
Eficácia da eletrolipólise na redução da adiposidade localizada: uma revisão integrativa
}

\author{
Electrolipolysis effectiveness in reducing localized adiposity: an integrative review
}

\author{
Gleice Braz de Camposa, Lucas Lima Ferreirab \\ a Fisioterapeuta. Pós-graduanda em Fisioterapia Dermato-funcional, Universidade Gama Filho, São Paulo, SP. \\ b Fisioterapeuta. Mestre em Fisioterapia, Universidade Estadual Paulista Júlio de Mesquita Filho (UNESP). Docente do Departamento de Fisioterapia da União das \\ Faculdades dos Grandes Lagos (UNILAGO), São José do Rio Preto, SP, Brasil.
}

RESUMO Introdução: A eletrolipólise é destinada ao tratamento das adiposidades e acúmulo de ácidos graxos localizados por meio da aplicação de pares de agulhas de acupuntura no tecido subcutâneo, ligadas a corrente de baixa frequência. Objetivo: Verificar a eficácia da eletrolipólise na redução da adiposidade localizada.

Materiais e Métodos: Foi realizada uma revisão integrativa de literatura, nas bases de dados SciELO, LILACS e PubMed, utilizando o cruzamento dos descritores physiotherapy, esthetics, abdomen, adiposity, lipolysis e do termo electrolipolysis. Foram incluídos ensaios clínicos com seres humanos maiores de 18 anos, nos idiomas português e inglês, no período de 2005 a 2015.

Resultados: Foram identificados cinco estudos cujo tamanho amostral variou entre oito a 16 sujeitos, totalizando 53 participantes, todos do sexo feminino, com idade entre 18 a 45 anos. A aplicação da eletrolipólise proporcionou desfechos significativos como reduções das medidas de adipometria de áreas como a região abdominal, glútea e em flancos.

Conclusão: A eletrolipólise na redução de medidas antropométricas, como a adiposidade localizada apresenta resultados favoráveis à sua aplicação.

Palavras-chave: modalidades de fisioterapia; estética; adiposidade; abdome.

Introduction: Electrolipolysis is for the treatment of fat deposits and accumulation of fatty acids found through the application of acupuncture needles pairs in the subcutaneous tissue, linked to low frequency current.

Objective: To determine the effectiveness of electrolipolysis in reducing localized adiposity.

Materials and Methods: An integrative literature review was conducted, in SCiELO, LILACS and PubMed databases using the crossing of descriptors: physiotherapy, aesthetics, abdomen, adiposity, lipolysis and electrolipolysis. Trials were included with adults over 18 years, in Portuguese and English, in the period between 2005-2015.

Results: We identified five studies in which the sample size ranged from eight to 16 subjects, totaling 53 participants, all female, aged 18-45 years. The application of electrolipolysis provided significant outcomes such as reductions in the measurements of areas such as the abdomen, buttocks and flanks.

Conclusion: Electrolipolysis presents favorable results in reducing anthropometric measures such as localized adiposity.

Keywords: physical therapy modalities; aesthetics; adiposity; abdomen. 


\section{INTRODUÇÃO}

No processo de evolução humana, o padrão de beleza passou por diversas mudanças e, devido à grande massificação das comunicações, surgiu uma tendência de padrão estético, no qual a adiposidade e as irregularidades da pele, como a celulite e as estrias, são pouco aceitas pela sociedade ${ }^{1}$. Contudo, a partir da definição do conceito de saúde como completo bem estar físico, psíquico e social, e não apenas como ausência de doença, foi possível compreender que o distúrbio estético representa uma ameaça à integridade emocional do indivíduo, resultante da alteração do esquema corporal e da autoestima ${ }^{2}$.

A adiposidade ou gordura localizada caracteriza-se pelo acúmulo de células gordurosas em partes específicas do corpo, causando um aumento na espessura e consistência do tecido adiposo. Esse excesso é resultado de um desequilíbrio entre consumo e demanda energética em alguma fase da vida do indivíduo em que há a dificuldade de manter os limites de gordura nos níveis desejados ${ }^{3}$. Na etiopatogenia da lipodistrofia gordurosa, temos como elementos causais mais frequentes o sedentarismo, o estresse, os antecedentes familiares, o tabagismo, alterações hormonais como a elevação do estrogênio, da aldosterona, da prolactina e da insulina, síndrome pré-menstrual, uso de anticoncepcionais, dismenorréias, algumas alterações ortopédicas sépticas e a patologia venosa ou linfática ${ }^{4}$.

A fisioterapia vem ampliando cada vez mais sua área de atuação, buscando sempre o equilíbrio entre saúde física e qualidade de vida. A fisioterapia dermato-funcional é uma área que vem acabando com o empirismo dos tratamentos estéticos, atuando assim na comprovação científica dos métodos e técnicas abordados para o tratamento de diversas patologias, uma vez que esse conhecimento proporciona uma abordagem direcionada à forma mais eficaz de tratamento para cada paciente, potencializando e assegurando resultados efetivos, sem causar riscos à saúde ${ }^{5,6}$.

Nesse contexto, a eletrolipólise é um recurso novo e promissor, que surge como opção terapêutica para o tratamento de diversas disfunções dermatológicas. A técnica é destinada ao tratamento das adiposidades e acúmulo de ácidos graxos localizados que, através da aplicação de pares de agulhas de acupuntura no tecido subcutâneo, ligadas a corrente de baixa frequência, atua diretamente no nível dos adipócitos e dos lipídios acumulados, produzindo sua destruição e favorecendo sua posterior eliminação ${ }^{7}$. O estímulo circulatório produz o ligeiro aumento de temperatura que se instala no local (efeito Joule) que contribui, em parte, para a instalação de uma vasodilatação, pois a corrente atua com estímulo direto nas inervações, promovendo uma ativação da microcirculação. Para Silva ${ }^{8}$ o estímulo circulatório produzido pela corrente elétrica tem grande importância na drenagem da área.

A frequência de aplicação oscila entre $5 \mathrm{~Hz}$ e $50 \mathrm{~Hz}$, sendo que a frequência de $25 \mathrm{~Hz}$ é mais eficaz para tratar alterações circulatórias e congestivas ${ }^{9,10}$. A ação hidrolipolítica da corrente se inicia com a estimulação do sistema nervoso simpático, provocando a liberação dos hormônios epinefrina e noraepinefrina pela supra-renal. Ambos se ligam aos receptores beta-adrenérgicos presentes na membrana celular dos adipócitos, provocando reações bioquímicas que vão culminar com a ativação da enzima triglicerídeo lipase sensível a hormônio, a qual hidrolisa os triacilgliceróis ${ }^{11}$.

Contudo, os níveis de evidência científica na especialidade de fisioterapia dermato-funcional ainda são muito baixos, apesar da grande aplicabilidade clínica dos recursos e técnicas nessa área. Buscas na literatura pertinente não identificaram estudos que sistematizassem as evidências atuais sobre a utilização de eletrolipólise nas disfunções estéticas mais comuns da atualidade como a adiposidade ou gordura localizada. Dessa forma, o presente estudo tem o objetivo de verificar a eficácia da eletrolipólise na redução da adiposidade localizada.

\section{MATERIAIS E MÉTODO}

O delineamento metodológico deste estudo caracterizouse por uma revisão integrativa da literatura.

\section{Estratégia de busca}

Foram identificados artigos científicos nacionais e internacionais selecionados a partir de consultas às bases de dados SciELO (ScientifC Eletronic Library Online), LILACS (Literatura Latino-Americana e do Caribe em Ciências da Saúde) e MedLine (Literatura Internacional em Ciências da Saúde) via PubMed no período de janeiro de 2005 a junho de 2015 e pesquisa foi realizada entre junho e agosto do corrente ano. Para a busca foram utilizados os cruzamentos das palavras-chave: fisioterapia (physiotherapy), estética (esthetics), abdome (abomen), adiposidade (adiposity), lipólise (lipolysis) por meio do operador boleando "and"; as quais foram definidas com base nos Descritores em Ciências da Saúde (DeCS) e seus correspondentes na língua inglesa Medical Subject Headings (MeSH). Além desses descritores, foi utilizado o termo eletrolipólise (electrolipolysis), que, mesmo não considerado um descritor pelo DeCS e MeSH, foi incluído em virtude de sua larga utilização como palavra-chave. Outra estratégia utilizada foi a busca manual nas listas de referências dos estudos selecionados para 
complementação da pesquisa. Todas as etapas da busca foram realizadas por somente um avaliador.

\section{Estratégia de seleção}

Para a seleção dos artigos, inicialmente foi realizada a triagem dos títulos relacionados ao tema em questão. Esta seleção foi baseada nos títulos que abordassem como ideia principal: a aplicação de eletroestimulação no tratamento de distúrbios estéticos. Ao final da busca, foram excluídos os títulos repetidos, já que esta foi realizada em diversas bases de dados. Em seguida, foi feita a leitura detalhada dos resumos dos artigos a fim de selecionar aqueles que abordassem exclusivamente a utilização da eletrolipólise como recurso terapêutico para redução de medidas corporais. Excluídos os resumos que não versavam sobre o tema, os textos completos foram avaliados e os que não se enquadravam nos critérios de exclusão foram inclusos como resultado final da busca.

\section{Critérios de inclusão e exclusão}

Foram incluídos estudos publicados nas línguas inglesa e portuguesa, com seres humanos de ambos os sexos, maiores de 18 anos, submetidos a tratamento para disfunções estéticas por meio da aplicação de eletrolipólise. Foram inclusos estudos longitudinais, randomizados e não randomizados. Resumos de dissertações ou teses acadêmicas, relatos de caso, trabalhos com animais e/ou crianças, revisões de literatura e estudos sem disponibilidade de texto completo disponível em algum sítio da internet foram excluídos.

\section{Análise dos dados}

Os dados foram analisados de forma qualitativa e apresentados na forma de tabelas com a descrição das seguintes características: autor, características da amostra, protocolo terapêutico e desfechos significativos.

\section{RESULTADOS}

A busca nas bases de dados pesquisadas resultou em 611 artigos, dos quais apenas cinco estudos clínicos ${ }^{12-16}$ foram incluídos segundo os critérios metodológicos estipulados (Figura 1).

Os artigos analisados tiveram suas principais informações compiladas na Tabela 1. Entre os cinco estudos identificados, verificou-se que a eletrolipólise foi aplicada como recurso em casos de adiposidade localizada com resultados satisfatórios na redução de medidas de adipometria e perimetria da região abdominal ${ }^{12-16}$. O tamanho amostral variou entre oito a 16 sujeitos, totalizando 53 participantes nos estudos selecionados, todos do sexo feminino, com idade entre 18 a 45 anos.

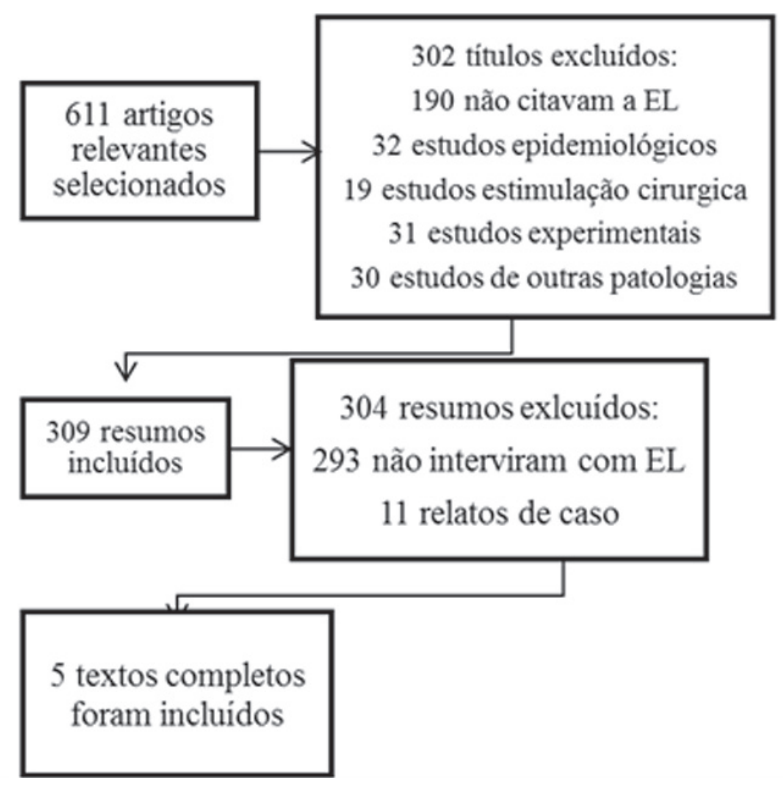

Figura 1. Fluxograma da estratégia de seleção dos artigos. EL: eletrolipólise.

Em relação às características da eletrolipólise aplicadas nos estudos analisados, verificou-se que não houve padronização de aparelhos, dos parâmetros de intervenção nem do tempo e número de sessões para aplicação. Contudo, foi possível observar que todos os estudos selecionados nesta revisão, demonstraram que a aplicação da eletrolipólise proporcionou desfechos significativos como reduções das medidas de adipometria de áreas como a região abdominal, glútea e em flancos.

No que tange aos métodos de avaliação após a aplicação da eletrolipólise, verificou-se que os estudos analisados utilizaram o índice de massa corporal (IMC), a perimetria e as dobras cutâneas em região abdominal, glútea e flancos, a relação cintura-quadril (C/Q) e a ultrassonografia de superfície.

\section{DISCUSSÃO}

O presente estudo analisou publicações recentes sobre a utilização de eletrolipólise como recurso para redução de medidas corporais, e observou que esta modalidade apresentou resultados positivos para sua aplicação em casos de adiposidade localizada, principalmente na região abdominal. Entretanto, a análise metodológica dos estudos identificados na busca, demonstrou a falta de padronização dos protocolos aplicados, assim como, as formas de avaliação dos resultados.

Rosa e Campos ${ }^{12}$ avaliaram os efeitos da eletrolipólise na redução da gordura abdominal localizada em mulheres jovens, comparando os valores de IMC, medidas de circunferência e medidas de dobras cutâneas antes e após o período de intervenção. O tratamento foi composto por 
Tabela 1. Características dos estudos que aplicaram eletrolipólise na adiposidade localizada.

\begin{tabular}{|c|c|c|c|}
\hline Autor & Características da amostra & Protocolo terapêutico & Desfechos significativos \\
\hline Rosa \& Campos ${ }^{12}$ & $\begin{array}{l}9 \text { pacientes, idade entre } 20 \text { a } 30 \text { anos, } \\
\text { IMC entre } 18,5 \mathrm{~kg} / \mathrm{cm}^{2} \text { e } 25 \mathrm{~kg} / \mathrm{cm}^{2} \text {, } \\
\text { dobras cutâneas abdominais medindo } \\
\text { até } 40 \mathrm{~mm} \text {, medidas de CA de até } \\
100 \mathrm{~cm} \text {, com gordura localizada na } \\
\text { região abdominal }\end{array}$ & $\begin{array}{l}10 \text { sessões de EL com duração de } 1 \text { hora } \\
\text { cada, uma vez por semana; A técnica } \\
\text { foi aplicada através de uma corrente } \\
\text { bifásica assimétrica, com largura de } \\
\text { pulso de } 300 \mathrm{~ms} \text {, frequência de } 5 \mathrm{~Hz} \text { e } \\
\text { a intensidade foi aumentada de acordo } \\
\text { com o limiar suportável pela paciente }\end{array}$ & $\begin{array}{l}\text { Foram observadas reduções nas } \\
\text { medidas de adipometria e perimetria da } \\
\text { região abdominal das pacientes, quando } \\
\text { comparadas as avaliações pré e } \\
\text { pós-intervenção }\end{array}$ \\
\hline Melo et al. ${ }^{13}$ & $\begin{array}{l}8 \text { pacientes, idade entre } 18 \text { e } 30 \text { anos, } \\
\text { divididas em } 2 \text { grupos: sedentárias e } \\
\text { não sedentárias }\end{array}$ & $\begin{array}{l}20 \text { sessões de EL com agulhas de } \\
\text { acupuntura dispostas } 6 \mathrm{~cm} \text { abaixo } \\
\text { da cicatriz umbilical, } 2 \text { vezes por } \\
\text { semana; intensidade de acordo com a } \\
\text { sensibilidade da paciente até o limite } \\
\text { de } 70 \mathrm{~mA}\end{array}$ & $\begin{array}{l}\text { Foi observada redução de medidas do } \\
\text { tecido adiposo em ambos os grupos, } \\
\text { havendo maior notoriedade no grupo } \\
\text { de sedentárias }\end{array}$ \\
\hline Mello-Carpes et al. ${ }^{14}$ & $\begin{array}{l}10 \text { voluntárias do sexo feminino, } \\
\text { selecionadas de forma intencional, idade } \\
\text { entre } 20 \text { e } 30 \text { anos, previamente hígidas }\end{array}$ & $\begin{array}{l}10 \text { sessões de EL, com duração de } 50 \\
\text { min por sessão, uma vez por semana; } \\
\text { frequência de } 30 \mathrm{~Hz} \text { e intensidade de } \\
\text { acordo com o limiar suportável pela } \\
\text { paciente }\end{array}$ & $\begin{array}{l}\text { Houve diminuição na perimetria e na } \\
\text { camada superficial de gordura analisada } \\
\text { por meio da ultrassonografia }\end{array}$ \\
\hline Azevedo et al. ${ }^{15}$ & $\begin{array}{l}10 \text { pacientes do sexo feminino, idade } \\
\text { entre } 18 \text { e } 25 \text { anos, selecionadas por } \\
\text { conveniência; subdivididas em } 2 \text { grupos: } \\
\text { G1 ( } 5 \text { pacientes) EL por acupontos e G2 } \\
\text { ( } 5 \text { pacientes) EL + AE }\end{array}$ & $\begin{array}{l}10 \text { sessões de EL por acupontos, com } \\
\text { duração de } 50 \text { minutos cada, com } \\
\text { frequência de } 15 \mathrm{~Hz} \text {, tempo ON de } 50 \mathrm{~ms} \text {, } \\
\text { tempo OFF de } 50 \mathrm{~ms} \text {, temporizador de } \\
20 \text { min para onda } \mathrm{C} \text { e } 10 \text { min para onde } \\
\text { E, intensidade regulada de acordo com a } \\
\text { tolerância da paciente; } \\
10 \text { sessões de } \mathrm{AE}: 30 \text { min de bicicleta } \\
\text { ergométrica após a EL, com velocidade } \\
\text { de } 20 \text { a } 25 \mathrm{~km} / \mathrm{h} \text { e } \mathrm{FC} \text { de } 60 \% \text { da FCM }\end{array}$ & $\begin{array}{l}\text { A EL foi eficaz na redução da adiposidade } \\
\text { abdominal, porém, associada à AE, } \\
\text { a redução de medidas de CA é mais } \\
\text { significativa }\end{array}$ \\
\hline Scorza \& Figueiredo ${ }^{16}$ & $\begin{array}{l}16 \text { mulheres, idade entre } 25 \text { e } 40 \text { anos, } \\
\text { multíparas e sedentárias, não submetidas } \\
\text { à dieta e AF durante o tratamento }\end{array}$ & $\begin{array}{l}10 \text { sessões de } \mathrm{EL}, 2 \text { vezes por semana; } \\
\text { frequência de } 30 \mathrm{~Hz} \text {, largura de pulso de } \\
250 \text { us, intensidade pela sensibilidade e } \\
\text { tolerância da voluntária, durante } 40 \text { min }\end{array}$ & $\begin{array}{l}\text { Observou-se redução do perímetro } \\
\text { abdominal em todos os quadrantes por } \\
\text { meio do exame ultrassonográfico }\end{array}$ \\
\hline
\end{tabular}

EL: eletrolipólise; IMC: índice de massa corporal; CA: circunferência abdominal; Hz: hertz; MS: milissegundos; mA: miliamperes; BMFG: biofotogrametria computadorizada; AE: atividade aeróbica; FC: frequência cardíaca; FCM: frequência cardíaca máxima; AF: atividade física; seg: segundos; RNM: ressonância nuclear magnética.

10 sessões com duração de uma hora, uma vez por semana, e os resultados apontaram que o nível de confiabilidade dos valores de perimetria e adipometria abdominal das participantes foi acima de $95 \%$. Quanto ao registro fotográfico as avaliadoras obtiveram $66,7 \%$ de acertos. Apenas, o IMC não apresentou alterações significativas. Os autores atribuem estes resultados ao objetivo primário da eletrolipólise, que é a redução de medidas, por meio da aplicação diretamente na área acometida utilizando finíssimas agulhas (modo percutâneo) ou através de eletrodos sobre a superfície cutânea (modo transcutâneo).

Outros autores ${ }^{13}$ avaliaram o efeito da eletrolipólise na redução de gordura localizada na região infra-abdominal, em pacientes que praticam atividade física (AF) e nas sedentárias, avaliando o IMC, a relação C/Q e por meio da perimetria de cintura (abaixo da última costela), da crista ilíaca, cinco cm e $10 \mathrm{~cm}$ acima da crista ilíaca. Foram realizadas 20 sessões, sendo duas vezes por semana, com oito agulhas de acupuntura conectadas a um aparelho de estimulação transcutânea de corrente de baixa frequência. Os resultados apontaram para redução de medidas do tecido adiposo em ambos os grupos, havendo maior notoriedade no grupo das sedentárias. O que pode ser explicado, segundo os autores, pelas características da estimulação utilizada, que proporciona semiondas quadradas positivas com pico negativo que estimulam os receptores nervosos ao mesmo tempo em que impedem a acomodação. Esses impulsos também incrementam o fluxo de sangue e eliminam os elementos de dejetos.

Mello-Carpes et al. ${ }^{14}$ analisaram a eficácia da utilização da eletrolipólise percutânea no tratamento de adiposidades localizadas na região abdominal e de flancos, utilizando como variáveis de comparação as medidas de perímetro corporal e a espessura da camada superficial de gordura por meio da ultrassonografia de imagem. O protocolo foi realizado uma vez na semana, totalizando 10 sessões, com aplicação de corrente percutânea (eletrodos de agulhas) numa frequência de $30 \mathrm{~Hz}$ e duração de $50 \mathrm{~min} /$ sessão. Os resultados demonstraram reduções significativas das variáveis de desfecho, fato atribuído às modificações morfofisiológicas que ocorrem nos adipócitos durante a aplicação da estimulação elétrica, dentre elas, o incremento do fluxo sanguíneo, aumentando o metabolismo celular e facilitando a queima de calorias. 
Outros autores ${ }^{15}$ analisaram os efeitos da eletrolipólise associada ou não ao trabalho aeróbico no tratamento da adiposidade abdominal grau I. O grupo que realizou trabalho aeróbico foi submetido a 30 minutos de bicicleta ergométrica, com velocidade de 20 a $25 \mathrm{~km} / \mathrm{h}$ e frequência cardíaca a $60 \%$ da frequência máxima, após as sessões de eletrolipólise. Após 10 sessões de tratamento, verificou-se redução média de $20 \%$ na prega cutânea abdominal. Porém, o resultado foi mais significativo no grupo submetido ao trabalho aeróbico após cada sessão de eletrolipólise com redução média de $23,88 \%$ na plicometria, chegando a $38,32 \%$ em uma das participantes. Os dados obtidos neste estudo são explicados pelo fato da eletrolipólise atuar na lipólise estimulando a ação do sistema nervoso simpático, ocorrendo a conversão intracelular de ATP em AMP cíclico.

Scorza et al. ${ }^{16}$ realizaram um ensaio clínico com objetivo de comparar os resultados obtidos na redução de adiposidade abdominal por meio da eletrolipólise com uso de estimulação nervosa transcutânea (TENS) no modo burst e normal. Foram realizadas 10 sessões, duas vezes por semana, em dias alternados, em 16 voluntárias, que durante o tratamento não foram submetidas à dieta e a atividade física. Os resultados apontaram para redução do perímetro abdominal em todos os quadrantes avaliados, em ambos os modos com o exame ultrassonográfico. Segundo os autores, o estímulo proporcionado pelo TENS, consegue reproduzir os mesmos efeitos fisiológicos obtidos com aparelhos clássicos de eletrolipólise, em virtude do efeito Joule, provocando modificações fisiológicas nos adipócitos, além do aumento de temperatura local que contribui para a instalação de vasodilatação com aumento do fluxo sanguíneo local.

Na análise dos métodos de avaliação após aplicação da técnica, pode-se observar que houve heterogeneidade de variáveis utilizadas para interpretar a eficácia da eletrolipólise. Tal achado limita possíveis inferências sobre os resultados obtidos, além disso, alguns dos métodos avaliativos utilizados não garantem fidedignidade à análise dos dados, quando aplicados por diferentes pesquisadores, uma vez que os estudos analisados não garantiram que as avaliações foram realizadas pelo mesmo pesquisador.

Os estudos analisados verificaram a efetividade da eletrolipólise na redução de medidas antropométricas em mulheres com adiposidade localizada em região abdominal. Todos estes ensaios obtiveram resultados favoráveis no que tange a redução de medidas corporais, reforçando a afirmação de que este recurso é mais uma modalidade disponível no arsenal terapêutico de profissionais que trabalham com a especialidade de fisioterapia dermato-funcional. Porém, não há consenso quanto ao tipo de aparelho ideal, bem como, os parâmetros, o tempo de intervenção em minutos e o número de sessões necessário para estes resultados.

Contudo, há que se ressaltar que a relevância e validade dos estudos analisados é baixa, pois existem inúmeros vieses nas publicações analisadas, tais como reduzido tamanho amostral, ausência de seguimento para avaliar se os efeitos são persistentes, falta de grupo controle para comparação adequada dos reais efeitos da técnica, entre outras. Estas restrições minimizam os níveis de evidência ora apresentados, sem, no entanto, invalidar as publicações sobre o tema.

Em suma, a análise metodológica dos estudos selecionados demonstrou que a eletrolipólise é um recurso para fisioterapeutas que atuam na especialidade de dermatofuncional, que pode apresentar bons resultados terapêuticos na redução do tecido adiposo localizado. Contudo, esta revisão pode apontar para falhas metodológicas importantes nos estudos encontrados na literatura pertinente, o que aponta para necessidade de novos ensaios clínicos com desenhos metodológicos adequados.

A presente revisão verificou que os estudos sobre eletrolipólise, aplicada como recurso na redução de medidas antropométricas, como a adiposidade localizada principalmente na região abdominal, apresentaram desfechos favoráveis à sua aplicação. Entretanto, os níveis de evidência dos estudos analisados são baixos e pode-se observar falta de padronização em termos de equipamentos e dos parâmetros para aplicação deste recurso terapêutico. Constatou-se, ainda, a escassez de estudos sobre o tema publicados até o momento, o que sucinta a necessidade de novas investigações com desenhos metodológicos específicos.

\section{REFERÊNCIAS}

1. Meyer PF, Lisboa FL, Alves MCR, Avelino MB. Desenvolvimento e aplicação de um protocolo de avaliação fisioterapêutica em pacientes com fibro edema geloide. Fisioter Mov. 2005;18(1): 75-83.

2. Querleux B, Cornillon C, Jolivet O, Bitton J. Anatomy and physiology of subcutaneous adipose tissue by in vivo magnetic resonance imaging and spectroscopy: relationships with sex and presence of cellulite. Skin Res Technol. 2002;8(2):11824. http://dx.doi. org/10.1034/j.1600-0846.2002.00331.x

3. Machado AFP. The effect of a cryotherapy gel in localized adiposity in young women. J Health Sci Inst. 2012;30(1):74-8.

4. Gironet N, Baulieu F, Giraudeau B, Machet L, Toledano C, Tiguemounine J, Lorette G, Vaillant L. Lymphedema of the limb: predictors of efficacy of combined physical therapy. Ann Dermatol Venereol. 2004;131(8-9):775-9. http://dx.doi.org/10.1016/S01519638(04)93759-3

5. Abe HT, Ferreira LL. Tratamento do fibroedema geloide com radiofrequência: revisão sistemática. Rev Pesq Fisioter. 2014;4(3):206-14. 
6. Ferreira LL, Fernandes C, Cavenaghi S. Fisioterapia dermatofuncional no fibro edema geloide: análise de periódicos nacionais. Rev Aten Saude. 2014;12(42):57-63.

7. Borges FS. Dermato-funcional: modalidades terapêuticas nas disfunções estéticas. São Paulo: Phorte; 2006.

8. Silva MT. Eletroterapia em estética corporal. São Paulo: Robe; 1997.

9. Assumpção AC, Souza A, Máximo I, Cardoso MC, Borges FS. Eletrolipólise (eletrolipoforese). In: Borges SF. Modalidades terapêuticas nas disfunções estéticas. São Paulo: Phorte; 2006. p. 217-25.

10. Guyton A. Tratado de fisiologia médica. Rio de Janeiro: Interamericana; 1998.

11. Zaragoza JR, Rodrigo P. Electroestética. Espanha: Nueva Estética; 1995.

12. Rosa JSR, Campos LG. Efeitos da eletrolipólise na redução de gordura abdominal em mulheres jovens. Rev Inspirar. 2014;6(5):13-8.
13. Melo NR, Monteiro FMAC, Pontes GAR, Mello SMB. Eletrolipólise por meio da estimulação elétrica transcutânea (TENS) na região abdominal em pacientes sedentárias e ativas. Fisioter Mov. 2012; 25(1):127-40.

14. Mello-Carpes PB, Stumpf T, Piccinini AM, Rosa PV. A eletrolipólise percutânea como possibilidade de diminuição da adiposidade em abdomen e flancos. Rev Biomotriz. 2012;6(2):94-104.

15. Azevedo CJD, Zanin EC, Tolentino TM, Cepeda CC, Busnardo VL. Estudo comparativo dos efeitos da eletrolipólise por acupontos e da eletrolipólise por acupontos associada ao trabalho aeróbico no tratamento da adiposidade abdominal grau I em indivíduos do sexo feminino com idade entre 18 e 25 anos. RUBS. 2008;1(2): 64-71.

16. Sorza FA, Figueirdo MM, Liao CO, Borges FS. Estudo comparativo dos efeitos da eletrolipólise com uso de TENS modo burst e modo normal no tratamento de adiposidade localizada abdominal. Ens Cienc. 2008;12(2):49-62. 